\title{
ÉDITORIAL
}

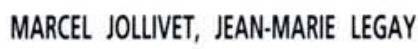

\section{Des catastrophes dites "naturelles" : questions actuelles}

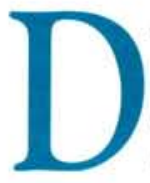

ans l'éditorial de son numéro 4 1993, Natures, Sciences, Sociétés a déjà évoqué la question des risques naturels.

Or voici que coup sur coup, deux "catastrophes naturelles" ont durement frappé deux des pays de la planète les plus riches et les plus développés techniquement: le 17 janvier 1995, c'était le Japon qui était endeuillé et ravagé par un tremblement de terre ; en février, cétait la Hollande qui devait faire face une nouvelle fois à l'invasion massive de l'eau. II faut certes prendre l'exacte mesure de ces événements et pour cela les rapprocher de ceux qui frappent périodiquement et de façon souvent bien plus dramatique les pays du tiers-monde.

Ce qui surprend, choque, inquiète, pose question dans le cas des Pays-Bas et du Japon, c'est qu'il s'agit de sociétés dites "évoluees", pourvues de moyens scientifiques et techniques sophistiqués, disposant de moyens matériels importants et d'équipements certifiés éprouvés.

Les risques d'inondations aux PaysBas, de tremblements de terre au Japon sont évidemment ceux qui sont les plus attendus et les plus craints et donc contre lesquels ces pays - "modernes" - cherchent à se prémunir en priorité en mobilisant pour cela au maximum savoirs, savoir-faire, techniques et moyens financiers. A travers ces accidents majeurs, c'est le mythe de la double confiance dans la technique et dans la manière de s'en servir qui est remis en cause.

En ce qui concerne la Hollande, on peut dire qu'après tout, les aménagements ont fait leur preuve, qu'il y a eu plus de peur que de mal et que ce qui est le plus remarquable en fin de compte, c'est la capacité de la société hollandaise à anticiper, puis à faire face, grâce à une organisation et une mobilisation sociales, ainsi qu'à un sang-froid collectif exemplaires : ceci renvoie bien sûr à une histoire nationale marquée par le rapport à l'eau et à ses dangers, à la menace permanente qu'elle représente pour le pays même. II n'en demeure pas moins qu'il y a eu doute sur la capacité des digues à tenir et il serait intéressant de se demander pourquoi un tel doute - qui semblerait devoir être exclu tant l'enjeu est grand - a pu être possible.

Pour ce qui est du Japon, les interrogations sont multiples et fondamentales. Elles portent tant sur l'efficacité des mesures préventives (le respect des normes antisismiques dans les constructions notamment) et du système d'alerte, que sur l'état de préparation du dispositif de sécurité civile destiné à faire face à l'état d'urgence. Là aussi une analyse s'imposerait.

Parler de "catastrophe naturelle" est en fait déformer la réalité. Si la cause est naturelle, sa transformation en catastrophe ne l'est pas forcément. On n'enregistre d'ailleurs certainement pas tous les accidents physiques graves se produisant à la surface de la terre, mais seulement ceux qui, dans les zones les plus habitées, s'accompagnent de nombreuses victimes et de dégâts matériels importants ; c'est ainsi qu'ils prennent rang de catastrophes dans l'expression des médias. Ce fait à soi seul mériterait aussi enquête et analyse.
Par ailleurs, si un tremblement de terre atteint une ville dont la plupart des constructions n'ont pas bénéficié de dispositifs antisismiques, celui-ci peut provoquer une catastrophe là où il aurait pu n'y avoir qu'un accident. Si une forte pluie atteint un bassin versant à la sortie duquel des régulations inintelligentes ont été mises en place ou sur lequel les transformations culturales ont rendu le sol à peu près imperméable, alors l'inondation en résultant en aval ne peut pas être acceptée comme étant naturelle.

L'histoire de la répartition du peuplement humain sur la terre a créé de multiples situations dans lesquelles des populations nombreuses sont affrontées à des phénomènes naturels de grande ampleur, représentant des risques majeurs pour les populations concernées. La question se pose de savoir dans quelles conditions de développement, avec quels coûts, mais aussi dans quelles limites techniques, le risque peut être réduit.

II serait d'un intérêt fondamental de procéder à des analyses de cas exemplaires comme ceux de la Hollande ou du Japon, pour mettre en évidence la façon dont les risques majeurs sont pris en compte, sur les plans tant scientifique que technique, économique, social et politique, dans les zones de grandes concentrations de population.

Ainsi la catégorie des "catastrophes naturelles" est un bon exemple de domaines où I'analyse interdisciplinaire que Natures, Sciences, Sociétés souhaite promouvoir s'avère particulièrement pertinente. 